\title{
Heat stress in the workplace
}

\author{
LILLY RAMPHAL, MD, MPH
}

$\mathbf{M}$ any work environments expose workers to extremely hot and humid conditions. The most vivid example of this is the firefighter, who inevitably must tolerate heat stress as part of the risks inherent to his or her job. Whereas the worker is expected to report imminent heat exhaustion before catastrophe occurs, ultimately the employer is held liable by the Occupational Safety and Health Administration (OSHA) for providing a safe work environment for its workers. Therefore, it is incumbent upon employers to screen workers for susceptibility and to rotate workers in and out of a hot work environment so as to prevent deleterious health outcomes, even fatalities.

Heat-related illness is a problem for many types of workers: metal smelters, outdoor construction and law enforcement workers, plastics manufacturing workers, landscaping and recreation maintenance personnel, staff in warehouses without air conditioning, cooks and kitchen workers, and athletes. A number of human factors contribute to a worker's susceptibility to heat stress, such as medical conditions, increasing age, overall level of fitness, presence of other metabolically stressful illnesses, the use of certain medications, dehydration, alcohol intake, and individual ability to acclimatize to extreme temperatures. Environmental factors that can contribute to heat stress besides high ambient temperature are low convection currents, high humidity, low evaporative loss, and high insulation levels around the body.

Acclimatization is defined as the time needed for physiological adaptation to extreme temperature changes. An average individual takes about 1 to 2 weeks to adapt to extreme cold or hot temperatures. Successful acclimatization occurs if the physiologic mechanisms of the cardiovascular, pulmonary, and renal systems interrelate effectively to adjust the body's core temperature by using evaporative heat loss or conservation. The process of acclimatization requires intact and responsive cardiovascular and renal systems.

The National Institute of Occupational Safety and Health has recommended that post-offer and surveillance examinations for heat stress include a complete history and physical examination to screen for relative or absolute contraindications to hot environments (1). These recommendations were made with the intent to screen workers who are more susceptible to heat stress before a catastrophic event occurs in the workplace. No specific examination exists to screen workers' susceptibility to hot environments. However, specific questions directed at screening for risk factors for heat stress can be included in the medical history to identify susceptible individuals. These questions should high-

\begin{tabular}{l} 
Table 1. Relative contraindications to heat stress exposure* \\
\hline The presence of chronic illness: \\
Renal disease \\
Thyroid disease \\
Diabetes or endocrine disorders \\
Heart disease or arrhythmias \\
Hypertension \\
Dehydration \\
Obesity \\
A history of previously documented heat-related illness \\
The use of certain medications: \\
Beta-blockers \\
Phenothiazines \\
Diuretics
\end{tabular}

*Source: reference 1.

light factors that are relative or absolute contraindications to prolonged exposure to hot environments.

Factors that may contribute to the tendency to develop heatrelated illnesses are considered relative contraindications to heat stress exposure (Table 1). Absolute contraindications are factors that are definitely known to contribute to heat-related illnesses (Table 2).

Relative contraindications to hot environments need to be assessed on a case-by-case basis, based on severity and lability of the worker's condition. For example, a severely hypertensive worker is more at risk than a moderately hypertensive worker. The person with severe hypertension is more likely to decompensate sooner than one with moderate hypertension.

Besides evaluating a worker with a complete history and physical examination, environmental monitoring with a wet bulb globe thermometer is recommended. This instrument measures ambient humidity, which has additive heat stress effects on human physiology.

Recommended ambient temperature limits vary, as the ambient humidity and convection factors vary. That is to say, as the

From BaylorWorx, Baylor Medical Center at Garland, Garland, Texas. Corresponding author: Lilly Ramphal, MD, MPH, 7501 Cedar Elm Drive, Irving, Texas 75063. 
Table 2. Absolute contraindications to heat stress exposure*

Pregnancy

Congestive heart failure

Unstable angina or arrhythmias

Severe hypertension

Renal failure

End-stage pulmonary disease

$>100.4^{\circ} \mathrm{F}$ core body temperature

Infertility

*Source: reference 1

humidity increases, the recommended temperature limits decrease. As the convection factors decrease, the recommended temperature limits decrease. Human factors such as an excessively rapid pulse ( $>90$ beats per minute) with slow recovery time after exercise (more than 3 minutes) and/or an elevated baseline core temperature $\left(>100.4^{\circ} \mathrm{F}\right)$ indicate a need for a rest period to allow for recovery to baseline before re-entry into the hot work area. Baseline measurements and exercise recovery time can serve as indicators whether or not a worker is fit to enter a hot environment at the beginning of the workday. Recommended alert limits and recommended exposure limit guidelines are encour- aged by OSHA. When workers are exposed to heat stress, biological monitoring with the 3 -minute pulse test recovery is recommended during the hottest time of the day or the hottest workplace location.

Absolute contraindications should be adhered to in workplaces. Workers with these risk factors or conditions should not be exposed to hot environments. If these risk factors are not identified, poor or fatal health outcomes may not be avoidable.

Exposure to a hot work environment requires that workers be continuously hydrated. Fluid loss through perspiration is genetically predetermined and proportional to the number of sweat glands per square inch of skin. Drinking water needs to be readily available to workers exposed to a hot work environment. Water misters are available that cool the skin continuously when workers voluntarily walk by. Construction crews at an outdoor worksite often use these.

In conclusion, the next time a company calls your office about a worker who has been overcome in a hot work environment, the patient history and environmental factors may be useful in helping you determine an etiology and whether that worker can return safety to that environment in the near or distant future, or maybe not at all.

1. NIOSH criteria documents for preventive practices of heat stress illness. $J$ Occup Environ Med 1996;38:689-692. 